SNUTP-98-113

hep-th/9810110

October, 1998

\title{
Sheets of BPS Monopoles and Instantons with Arbitrary Simple Gauge Group
}

\author{
Kimyeong Le曰 \\ Physics Department and CTP, Seoul National University, Seoul 151-742, Korea
}

\begin{abstract}
We show that the BPS configurations of uniform field strength can be interpreted as those for sheets of infinite number of BPS magnetic monopoles, and found that the number of normalizable zero modes per each magnetic monopole charge is four. We identify monopole sheets as the intersecting planes of D3 branes. Similar analysis on self-dual instanton configurations is worked out and the number of zero modes per each instanton number is found to match that of single isolated instanton.
\end{abstract}

${ }^{1}$ Electronic Mail: kimyeong@phya.snu.ac.edu 
There has been some interest in BPS field configuration with uniform magnetic field [1]. This background field exerts no force on isolated BPS monopoles as the repulsive magnetic force cancels the attractive Higgs force. Thus there is a possibility of BPS configuration of an isolated monopole in such background, which has been explored recently [2]. Besides this, there has been a long standing interest in similar uniform configuration of four dimensional self-dual equation of YangMills theories [3]. This configuration is known to be stable and the zero modes of this configuration has been explored to understand the quantum correction to the effective action.

In this paper we show that the BPS configuration with uniform magnetic field can be interpreted as a two-dimensional sheet made by infinite number of BPS magnetic monopoles lying on a plane. By studying zero modes, we show that the number of normalizable zero modes per unit magnetic flux is four, identical to that for an isolated monopole. Along the planar direction of the monopole sheet, the zero modes are given by the wave function of the lowest Landau level, so that the monopole sheet appears a quantum phase space or noncommutative plane. By considering the case of the general gauge group which is maximally broken, we show that magnetic monopole sheets exist for each pair of positive and negative root vectors. Again for unit magnetic monopole flux, there are four normalizable zero modes. Especially for $\mathrm{SU}(\mathrm{N})$ gauge group, the magnetic monopole sheets can be identified as intersecting planes of D3 branes in type IIB string theory. For the instanton case, the zero modes are well-known. What is new here is that the center position of normalizable zero modes is pointed out to live on four dimensional quantum phase space. In theories with general gauge group, we also show that the number of normalizable zero modes per unit Pontryagin index is identical to four times the dual Coxeter number, $4 c_{2}(G)$, which has been obtained from the index theorem for isolated instantons [- 4 and from the counting the number of constituent monopoles of instantons on $R^{3} \times S^{1}[5]$.

In a Yang-Mills Higgs theory where $S U(2) \rightarrow U(1)$, the field configuration for single monopole is spherically symmetric and has a core region of size $1 / m_{W}=1 / v$ with coupling constant $e=1$. Inside the core the gauge field for the massive $W$ bosons is nonzero, but vanishes exponentially outside the core. When we put $N>0$ BPS magnetic monopoles as close as possible, the core configuration becomes complicated. For two monopoles, the core is a torus [6], for three monopoles, the core is tetrahedral [0], and so on.

For large $N$, the shape of the core shape becomes presumably more involved. Whatever shape it takes, one may ask what is the rough size of the core region. The answer can be found readily by considering the asymptotic behavior of the Higgs field. Since the Higgs field approaches $(v-N / r)$ 
asymptotically, the natural scale of the core size is

$$
r_{\text {core }} \sim N / v=N / m_{W}
$$

Now imagine configurations with an increasing number of magnetic monopoles. A simple way is to put magnetic monopoles along a line with an equal distance $d$, which, say, is much greater than $1 / m_{W}$. We know this configuration cannot be axially symmetric as there exits a relative orientation between any two monopoles. Since the minimum core size (11) is less than the size of the configuration, monopole cores seem to be separated. However there exits an additional logarithmic divergence in the monopole core size. To see this, consider the value of the Higgs field for a fixed point in large $N$ limit. It would be roughly of order

$$
|\phi|-v \sim \sum_{n=1}^{N}-\frac{1}{n d} \sim-\frac{1}{d} \ln N .
$$

Thus, the core of magnetic monopoles will eventually merge together when $N \sim e^{d v}$. The large $N$ limit can be taken only if we increase $v$ simultaneously. Otherwise, we will end up with just symmetric phase. This picture is consistent with another view of lined magnetic monopoles. Far away from the line, the $U(1)$ magnetic field will fall off like $1 / r$ with $r$ being the distance from the line. This means the Higgs field will increase like $\log r$.

Now imagin putting $N$ monopoles on a two dimensional square lattice of lattice size $d$. With one monopole on each lattice site, they occupy a square of size $\sqrt{N} d$. Since the core size (1) grows linearly with $N$, the cores of these monopoles will start to overlap for $N \sim(v d)^{2}$ and individual monopoles becomes indistinguishable. As in the linear configuration, we can take the infinite $N$ limit of the planar configuration only if we take $v=\infty$ simultaneously. The magnetic field far from the plane is approximately uniform, which in turn implies that the Higgs field grows linearly. There may be several BPS field configurations which can be interpreted as those corresponding the sheet of BPS magnetic monopoles. It appears hard to find any of such BPS configurations. In this paper, we argue that the well-known homogeneous BPS configuration

$$
B_{i}^{a}=D_{i} \phi^{a}=b \delta_{a 3} \delta_{i 3}
$$

is one of such configurations. It is obvious in retrospective that it should be so. The corresponding Higgs and gauge fields are

$$
\begin{aligned}
\bar{\phi}^{a} & =b \delta^{a 3}\left(z-z_{0}\right), \\
\bar{A}_{i}^{a} & =\frac{b}{2} \delta^{a 3}(-y, x, 0)
\end{aligned}
$$


in the symmetric gauge. Note that the origin of the $x, y$ plane is not special as there is a translation symmetry on that plane. However, the plane $z=z_{0}$ is very special as the gauge symmetry is restored only on that plane. The naive $W$ boson mass increases as one moves away from the plane. Also the gauge invariant $U(1)$ magnetic field $B_{i}^{a} \hat{\phi}^{a}=b \operatorname{sign}\left(z-z_{0}\right)$ changes the sign as one crosses the plane. Both of them implies that the plane could be interpreted as the sheet of BPS magnetic monopoles. The individual characteristics of magnetic monopoles disappear, and so do the nonabelian characteristics of the core region. Thus there is no core region at all. Still, there emerges some individuality of monopole as we will see. As single magnetic monopole carries the magnetic flux $4 \pi$ and the magnetic fields of opposite sign come out from the both sides of the sheet with strength $b$, we can assign the magnetic monopole number density per unit area to be

$$
n_{\text {monopole }}=\frac{b}{2 \pi} \text {. }
$$

We note that in the $\mathrm{SU}(2)$ case only one sheet is allowed.

The interesting question is then whether we can take out single BPS magnetic monopole away from the sheet. While we do not know the answer for this question, we can begin by exploring what are the normalizable zero modes of the magnetic monopole sheet.

The linear fluctuations $\delta A_{i}^{a}, \delta \phi^{a}$ satisfy the linearized BPS equations

$$
\epsilon_{i j k}\left(\partial_{j} \delta A_{k}^{a}+\epsilon^{a b c} \bar{A}_{j}^{b} \delta A_{k}^{c}\right)=\left(\partial_{i} \delta \phi^{a}+\epsilon^{a b c} \bar{A}_{i} \delta \phi^{c}\right)-\epsilon^{a b c} \bar{\phi}^{b} \delta A_{i}^{c},
$$

and the background gauge condition

$$
\left(\partial_{i} \delta A_{i}^{a}+\epsilon^{a b c} A_{i}^{b} \delta A_{i}^{c}\right)+\epsilon^{a b c} \bar{\phi}^{b} \delta \phi^{c}=0 .
$$

The background gauge is implied by the Gauss law satisfied by the slowly moving monopole in the $A_{0}$ gauge. The fluctuations $\delta A_{i}^{3}, \delta \phi^{3}$ along unbroken abelian direction are independent of the background and non-normalizable. They correspond to the change in the position and orientation of the magnetic monopole sheet.

For the study of fluctuations along broken symmetry direction, we introduce

$$
\begin{aligned}
& W_{i}=\frac{1}{\sqrt{2}}\left(\delta A_{i}^{1}+i \delta A_{i}^{2}\right), \\
& W_{4}=\frac{1}{\sqrt{2}}\left(\delta \phi^{1}+i \delta \phi^{2}\right),
\end{aligned}
$$

which describe to massive $W$ bosons in the ordinary case. The linearized BPS equations and the background gauge condition become

$$
\begin{aligned}
& \epsilon_{i j k} D_{j} W_{k}=D_{i} W_{4}-D_{4} W_{i}, \\
& D_{i} W_{i}+D_{4} W_{4}=0,
\end{aligned}
$$


where

$$
\begin{gathered}
D_{i}=\partial_{i}-i V_{i}, \quad V_{i}=\frac{b}{2}(-y, x, 0), \\
D_{4}=\partial_{4}-i V_{4}, \quad V_{4}=b\left(z-z_{0}\right) .
\end{gathered}
$$

Of course the fluctuations considered here are independent of $x^{4}$ and so $\partial_{4}=0$.

These equations can be easily solved. There are two independent families of solutions:

$$
\begin{aligned}
& W_{1}=-i W_{2}=c_{1} e^{\frac{i b}{2}\left(x_{0} y-y_{0} x\right)-\frac{b}{4}\left(\left(x-x_{0}\right)^{2}+\left(y-y_{0}\right)^{2}-\frac{b}{2}\left(z-z_{0}\right)^{2}\right.}, \\
& W_{3}=-i W_{4}=c_{2} e^{\frac{i b}{2}\left(x_{0} y-y_{0} x\right)-\frac{b}{4}\left(\left(x-x_{0}\right)^{2}+\left(y-y_{0}\right)^{2}-\frac{b}{2}\left(z-z_{0}\right)^{2}\right.},
\end{aligned}
$$

where $c_{1}$ and $c_{2}$ are two independent constants. Then these two families and their complex conjugate form four independent families of zero modes. Along the $z$ direction, the zero modes are given by the ground state wave function of harmonic oscillator, which is concentrated on the monopole sheet $z=z_{0}$. This is due to the fact that the mass of $W$ boson is zero on that sheet and increases linearly away from that. Along the $x-y$ direction, the zero modes are given by the wave function of the lowest Landau level. Since we have chosen the symmetric gauge for the uniform magnetic field, the wave function can be chosen to be concentrated on the position $\left(x_{0}, y_{0}\right)$.

Now let us recall the well known physics of the Landau levels. The conserved $x$ and $y$ translations of the wave function do not commute each other, making the $x-y$ plane a quantized phase space, or noncommutative plane. The conserved generators of translations are $\pi_{x}=-i \partial_{x}-e B y / 2$ and $\pi_{y}=-i \partial_{y}+e B x / 2$, satisfying the commutation relation,

$$
\left[\pi_{x}, \pi_{y}\right]=i e B
$$

Thus, the parameters $x_{0}, y_{0}$ appearing in the wave function overcount the number of independent ground wave function. This can be seen easily by choosing the Landau gauge and compactifying the $x-y$ plane. The degeneracy is not infinite in spite that $x_{0}, y_{0}$ are continuous. Rather, there is a minimum area $2 \pi / e B$ per one state. Thus, in our notation $e=1, B=b$, the number density of independent ground states per unit area is given by

$$
n_{\text {landau }}=\frac{b}{2 \pi} \text {. }
$$

In terms of the magnetic length $l_{b}=1 / \sqrt{b}$, one can understand the quantum cell $2 \pi l_{b}^{2}$ as the size of the minimum quantum volume of the phase plane. Also the total magnetic flux in this quantum cell is the Dirac quantum flux $2 \pi$, which is the minimum magnetic flux one can put consistently in the compactified $x-y$ space. 
Coming back to our magnetic monopole problem, the number density of the zero modes is four

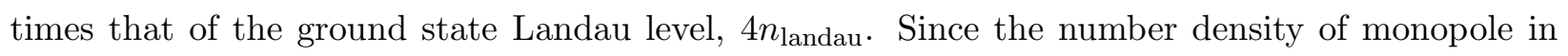
Eq. (6) is identical to the $n_{\text {landau }}$, the number of zero modes per unit magnetic monopole becomes four. This is exactly identical to the number of zero modes for single BPS monopole in $\mathrm{SU}(2)$ gauge group. For one monopole, three of them account its position and one does for its internal phase angle. In our case monopoles are completely dislocalized on the sheet, but we can imagine figuratively single quantum cell as one monopole. As our analysis is at linear level, we do not know exactly how these zero modes will develop nonlinearly. It would be interesting to find the full nonlinear distortion of the monopole sheet and the geometry of the infinite dimensional moduli space of zero modes. Due to the fact that the $x_{0}, y_{0}$ space form noncommutative plane, the moduli space itself may be noncommutative.

Let us now generalize the above consideration to Yang-Mills Higgs theory of arbitrary simple gauge group $G$ of rank $r(G)$ and dimension $d(G)$. We split the generators of the Lie algebra to the Cartan subalgebra and the raising and lowering operators. We normalize the generators so that in the adjoint representation

$$
\begin{aligned}
& \operatorname{tr}\left(H_{a} H_{b}\right)=c_{2}(G) \delta_{a b}, \\
& \operatorname{tr}\left(E_{\alpha}^{\dagger} E_{\beta}\right)=c_{2}(G) \delta_{\alpha \beta},
\end{aligned}
$$

where $a, b=1, \ldots, r(G)$. The normalization factor $c_{2}(G)$ is the quadratic Casimir for the adjoint representation and becomes the dual Coxeter number when the longest root vectors is normalized to have length one. (See for example Ref. [4) Among the commutation relations, ones we need are

$$
\left[\mathbf{H}, E_{\alpha}\right]=\boldsymbol{\alpha} E_{\alpha}
$$

where $\mathbf{H}$ and $\boldsymbol{\alpha}$ are $r(G)$ dimensional vectors. The eigenvalues of the matrix $H_{a} H_{b}$ consist of $\boldsymbol{\alpha}_{a} \boldsymbol{\alpha}_{b}$ for all roots and so Eq. (16) implies an identity

$$
\sum_{\boldsymbol{\alpha}} \boldsymbol{\alpha}_{a} \boldsymbol{\alpha}_{b}=c_{2}(G) \delta_{a b}
$$

The BPS equation in this general group becomes

$$
B_{i}=D_{i} \phi
$$

where

$$
\begin{array}{r}
B_{i}=\frac{1}{2} \epsilon_{i j k}\left(\partial_{j} A_{k}-\partial_{k} A_{j}-i\left[A_{j}, A_{k}\right]\right), \\
D_{i} \phi=\partial_{i} \phi-i\left[A_{i}, \phi\right] .
\end{array}
$$


The uniform solution of the BPS equation can be chosen to lie in the Cartan subalgebra,

$$
B_{i}=D_{i} \phi=\mathbf{b}_{i} \cdot \mathbf{H}
$$

where $\mathbf{b}_{i}$ are $r$-dimensional vectors for each $i=1,2,3$. The corresponding field configuration in the symmetric gauge is

$$
\begin{aligned}
\bar{A}_{i} & =-\frac{1}{2} \epsilon_{i j k} x^{j} \mathbf{b}_{k} \cdot \mathbf{H} \\
\bar{\phi} & =x^{i} \mathbf{b}_{i} \cdot \mathbf{H}+\mathbf{h} \cdot \mathbf{H} .
\end{aligned}
$$

There are still remaining gauge transformations: the unbroken $U(1)^{r(G)}$ transformations and the Weyl reflections which shuffle the generators in the Cartan subalgebra.

The linearized BPS equations for $\delta A_{i}, \delta \phi$ in this background is

$$
\epsilon_{i j k}\left(\partial_{j} \delta A_{k}-i\left[\bar{A}_{j}, \delta A_{k}\right]\right)=\partial_{i} \delta \phi-i\left[\bar{A}_{i}, \delta \phi\right]-\partial_{4} \delta A_{i}+i\left[\bar{\phi}, \delta A_{i}\right]
$$

The background gauge condition is

$$
\partial_{i} \delta A_{i}-i\left[\bar{A}_{i}, \delta A_{i}\right]+\partial_{4} \delta \phi-i[\bar{\phi}, \delta \phi]=0
$$

Again, as there is no $x^{4}$ dependence, $\partial_{4}=0$. As in the $S U(2)$ case, the background field $\bar{A}_{i}, \bar{\phi}$ lies on the Cartan subalgebra and the equations are linear, and so the linear fluctuations along the generators do not mix. The fluctuations $\delta A_{i}, \delta \phi$ along unbroken gauge groups is independent of the background fields and unnormalizable. They are associated with $\delta \mathbf{b}_{i}$ and $\delta \mathbf{h}$.

For the study of fluctuations lying along a raising operator $E_{\boldsymbol{\alpha}}$, we introduce $W_{\mu}$ such that

$$
\delta A_{i}=W_{i} E_{\alpha}, \delta \phi=W_{4} E_{\alpha} .
$$

The $W_{\mu}$ equations become identical to those in Eq. (11) if we rotate the coordinate so that $\mathbf{b}_{i} \cdot \boldsymbol{\alpha}$ points to $\hat{z}$ direction and put

$$
b=\left|\mathbf{b}_{i} \cdot \boldsymbol{\alpha}\right|, \quad z_{0}=-\mathbf{h} \cdot \boldsymbol{\alpha} .
$$

Then the solution will be identical as those in Eq. (13). Since zero modes are confined on the plane

$$
x^{i} \mathbf{b}_{i} \cdot \boldsymbol{\alpha}+\mathbf{h} \cdot \boldsymbol{\alpha}=0,
$$

we interpret this plane as the $\boldsymbol{\alpha}$ monopole sheet. Note that this plane is invariant under $\boldsymbol{\alpha} \rightarrow-\boldsymbol{\alpha}$. Similarly to the $S U(2)$ case, the number density of $\boldsymbol{\alpha}$ monopole is

$$
n_{\boldsymbol{\alpha}}=\frac{\left|\mathbf{b}_{i} \cdot \boldsymbol{\alpha}\right|}{2 \pi},
$$


and the number of zero modes per unit quantum cell or BPS monopole is again four.

When there is unbroken nonabelian subgroup so that the planes $(\sqrt[29]{29})$ do not overlap, the number of monopole sheet is that of positive roots $(d(G)-r(G)) / 2$. Contrast to the case of finite number of magnetic monopoles where there is fundamental magnetic monopoles corresponding to simple roots [8], all monopole sheets in our case are in equal footing.

The D-brane picture of $N=4$ dimensional Yang-Mills theory in four dimension helps to understand our result [10]. Monopoles appear as D-strings connecting parallel D3 branes in type 2B string theory. Following the work by Callan and Maldacena [9] these D-strings can be regarded as continuous deformation of D3 branes. Our work suggests that as the number of magnetic monopoles increases to infinite, something drastic happens. The D3 branes get tilted and appear to intersecting each other. Since D3 branes are self-dual, there is no distinction between intersecting and contacting of two D3 branes. Since the field configuration is self-dual, this configuration of interacting D3 branes should be supersymmetric. In the $\mathrm{SU}(2)$ case there is an obvious duality between the $z$ direction and $\phi$ direction in the D-brane picture, but it is not obvious in the field theory picture. In $\mathrm{SU}(\mathrm{N})$ case, we can rewrite the Higgs field in Eq. (24) in the $N$ dimensional Hermitian matrix form. Its $i$-th diagonal component then indicates the position of the $i$-th D3 brane. Thus, one can identify each monopole sheet associated with a given $\operatorname{root} \boldsymbol{\alpha}=\mathbf{e}_{i}-\mathbf{e}_{j}$ with the plane of intersection between the $i$-th and $j$-th D3 branes.

Let us now change our attention to the instanton case. Instantons are the solutions of self-dual equations,

$$
B_{i}=F_{i 4}
$$

in Euclidean four dimensional Yang-Mills theory. The self-dual configuration with homogeneous field strength is

$$
B_{i}=F_{i 4}=\mathbf{b}_{i} \cdot \mathbf{H}
$$

The Pontryagin index density of this configuration is

$$
n_{\text {Pont }}=\frac{1}{32 \pi^{2} c_{2}(G)} \operatorname{tr}\left(F_{\mu \nu} \tilde{F}_{\mu \nu}\right)=\frac{\mathbf{b}_{i} \cdot \mathbf{b}_{i}}{8 \pi^{2}} .
$$

Once we choose the symmetric gauge for $B_{i}$ and the Landau gauge for $F_{i 4}$ so that $\partial_{4} A_{i}=0$, the background field is identical to those in Eq. (24) with $A_{4}=\phi$. Contrast to the magnetic monopole case, the plane (29), which is now a three volume, is not special any more as $\mathbf{h} \cdot \boldsymbol{\alpha}$ can be changed by the unbroken gauge transformations. Rather for each $\mathbf{b}_{i} \cdot \boldsymbol{\alpha}, B_{i}$ is the magnetic field through the two-dimensional plane defined by $x^{i} \mathbf{b}_{i} \cdot \boldsymbol{\alpha}=0$ and $x^{4}=0$, and $F_{i 4}$ is the magnetic field through the two-dimensional $x^{i} \sim \mathbf{b}_{i} \cdot \boldsymbol{\alpha}$ and $x^{4}$ plane. 
The linear fluctuation equations for $\delta A_{\mu}$ will be identical to Eq. (25), once we allow the $x^{4}$ dependence. The background gauge condition is then identical to Eq. (26). The fluctuations along the unbroken abelian direction is again independent of the background and nonrenormalizable. They changes the parameters $\mathbf{b}_{i}$ and $\mathbf{h}$.

On the other hand, for the fluctuations along a raising operator $E_{\boldsymbol{\alpha}}$, we use Eq. (27) to introduce $W_{\mu}$. With the $x^{4}$ dependence, $e^{i p^{4} x^{4}}$, and a spatial rotation such that $\mathbf{b}_{i} \cdot \boldsymbol{\alpha}$ lying along the $z$ direction, the zero mode solutions are identical to monopole case (13) once we use Eq. (28) and replace $z_{0}$ by $z_{0}+p^{4} / b$. This shows that the zero mode is not confined on the plane (29). Rather the four zero mode solutions are the product of two wave functions of the lowest Landau level, one on the $x-y$ plane and another on the $z-x^{4}$ plane. First is written in the symmetric gauge and second is in the Landau gauge. As far as these zero modes are concerned, the translation along the $x$ direction and those along the $y$ direction do not commute. This makes again the $x-y$ plane a phase space or a quantum plane with unit quantum cell of area $2 \pi / b$. Similarly the $z-x^{4}$ plane becomes a quantum plane of unit quantum cell of area $2 \pi / b$. Thus on the $R^{4}$ space the number density of zero modes for each positive root $\boldsymbol{\alpha}$ is

$$
n_{\boldsymbol{\alpha}}=4\left(\frac{\mathbf{b}_{i} \cdot \boldsymbol{\alpha}}{2 \pi}\right)^{2}
$$

The number density of zero modes from all positive roots is the sum

$$
n_{\text {zero }}=\sum_{\boldsymbol{\alpha}>0} n_{\boldsymbol{\alpha}}=\frac{\mathbf{b}_{i} \cdot \mathbf{b}_{i}}{2 \pi^{2}} c_{2}(G),
$$

where Eq. (19) is used. The number of zero modes per unit Pontryagin index is then

$$
\frac{n_{\text {zero }}}{n_{\text {Pont }}}=4 \times c_{2}(G) .
$$

This number is exactly identical to what is obtained from the index theorem [4] and also from the consideration of constituent monopoles of calorons [5].

Instantons appear as the bound states of D0 branes on overlapped D4 branes of the type 2A theory. Our case will be the limit where the number of D0 branes is infinite. There is a work recently on this limit [11]. The D-brane picture of our homogeneous solution for instanton is much less clear than that for monopoles. While we assume that homogeneous self-dual configuration can be obtained by arranging hte infinite number of instantons right, we do not know how they are put together for the homogeneous configuration.

In this paper we explored the homogeneous BPS field configuration as the sheets of infinite number of BPS magnetic monopoles. The normalizable zero modes is confined at the magnetic 
monopole sheet, even though their position on the plane is not localized. Their position on plane are described by the quantized phase space or noncommutative two-plane. We showed that the number of normalizable zero modes per unit magnetic monopole charge is four. For instantons, the number of normalizable zero modes per unit Pontryagin number is identical to that of single instanton without uniform background. The position space $R^{4}$ of the normalizable zero modes become noncommutative four space. We also discussed the D-brane pictures of these homogeneous configurations.

There are several questions arising naturally. First question is whether single magnetic monopole can be separated from the infinite sheet. Forementioned work on the $S U(2)$ case by C. Lee and Q.H. Park suggests that it cannot be done without singular behavior in other side of the monopole sheet. We think this needs a further clarification. On the other hand, there exists a remarkable Minkowski solution [12] in the instanton case. It describes the instanton lump in the uniform background.

When $\mathbf{b} \cdot \boldsymbol{\alpha}=0$ for a root $\boldsymbol{\alpha}$, one can embed the corresponding $S U(2)$ monopole or instanton solutions. In the monopole case with the $S U(N)$ gauge group, two D3 branes are parallel and any finite number of D string can be inserted. In the instanton case, we do not know what arrangement of D4 and D0 makes such a special case. Note that the number of zero modes per unit Pontryagin index is independent whether $\mathbf{b}_{i} \cdot \boldsymbol{\alpha}=0$ or not.

Second, it would be interesting to find out the moduli space dynamics of zero modes of these monopole and instanton sheet. The moduli space would be now infinite dimensional. As the position space of these zero modes are noncommutative, the moduli space may be noncommutative and the low energy Lagrangian could be some sort of a field theory of noncommutative variables.

Third, let us ask how $S U(2)$ monopole or instanton sheets can be obtained from ADHM and Nahm formalisms [13]. Since there are infinite number of monopoles and instantons, the $S U(\infty)$ group appears naturally. Ward's work 14] on Nahm's equation should be relevant to the magnetic monopole sheet. But we do not know the corresponding ADHM formalism or the ADHMN construction of solutions. In addition, for instanton case, our work seems to be related to the recent work on the noncommutative geometry [15]. It remains to be clarified.

\section{Acknowledgments}

I thank Bum-Hoon Lee, Choonkyu Lee, Nick Manton, Soo-Jong Rey, and Erick Weinberg for useful discussions. This work is done partly while I was visiting the SNU-CTP during the 1998 summer. This work was also supported by KOSEP 1998 Interdisciplinary Research Program and 
SRC program of SNU-CTP, and Ministry of Education BSRI 98-2418. 


\section{References}

[1] D. Bak, C. Lee, and K. Lee, Phys. Rev. D57 (1998) 5239.

[2] C. Lee and Q.H. Park, Exact BPS monopole solution in a self-dual background, Seoul N. University Preprint (1998), SNUTP-98-096.

[3] L.S. Brown and W.I. Weisberger, Nucl. Phys. B157 (1979) 285; H. Leutwyler, Nucl. Phys. B179 (1981) 129; P. Schwab, Phys. Lett. 109B (1982) 47; G. 't Hooft, Comm. Math. Phys. 81 (1981) 267.

[4] C.W. Bernard, N.H. Christ, A.H. Guth and E.J. Weinberg, Phys. Rev. D16 (1977) 2967.

[5] K. Lee, Phys. Lett. B426 (1988) 323.

[6] R.S. Ward, Phys. Lett. 102B (1981) 136; M.F. Atiyah and N.J. Hitchin, The Geometry and Dynamics of Magnetic Monopoles (1988) (Princeton, NJ: Princeton University Press).

[7] H.J. Hitchin, N.S. Manton and M.K. Murray, Nonlinearity 8 (1995) 661; C.M. Houghton and P.M. Sutcliffe, Nucl. Phys. B464 (1996) 59.

[8] E.J. Weinberg, Nucl. Phys. B167 (1980) 500.

[9] C.G. Callan and J.M. Maldacena, Nucl. Phys. B513 (1998) 198.

[10] E. Witten, Nucl. Phys. B460 (1996) 335; A.A. Tseytlin, Nucl. Phys B469 (1996) 51; M.B. Green and M. Gutperle, Phys. Lett. B377 (1996) 28.

[11] E. Keski-Vakkuri and P. Kraus, Nucl. Phys. B510 (1998) 199.

[12] P. Minkowski, Nucl. Phys. B177 (1981) 203.

[13] M.F. Atiyah, N.J. Hitchin, V.G. Drinfeld and Yu.I. Mannin, Phys. Lett. 185B (1978) 185; W. Nahm, in Monopoles in quantum field theory, ed. N.S. Craigie et al. (World Scientific, Singapore, 1982).

[14] R.S. Ward, Phys. Lett. B234 (1990) 81.

[15] N. Nekrasov and A. Schwarz, Instantons and noncommutative $R^{4}$, and $(2,0)$ superconformal six-dimensional theory, hep-th/9802068 